\section{Chronic inflammatory arthritis following checkpoint inhibitor therapy for cancer: game changing implications}

\author{
Leonard Calabrese (1) , ${ }^{1}$ Xavier Mariette ${ }^{2,3}$
}

The use of immune checkpoint inhibitor (ICI) therapy for cancer is now a pillar of oncological therapeutics and growing, with an estimated $43.5 \%$ of all tumours falling within current labelling indications for use. ${ }^{1}$ Eventually as the accessibility to ICI therapy increases, these data have staggering implications, given that an estimated number of new cancers in Europe and the USA exceeds 5 million individuals yearly. ${ }^{2}$ As a byproduct of this tidal wave of newly exposed patients to various forms of immunotherapy with estimates that $10 \%-20 \%$ or more who may develop serious immune related adverse events (irAEs), ${ }^{3}$ it is inevitable that the evaluation and care of such patients will pose a challenge to existing healthcare systems and likely create a space for a new specialty to manage such. From a rheumatological perspective, let us now consider that an estimated 3\%-7\% of ICI exposed patients may develop inflammatory arthritis (IA), ${ }^{45}$ making it seem inevitable that ICI associated IA will become ever more commonplace, giving us pause to ask ourselves what our current understanding of this disorder is and how prepared we are to manage it.

These irAEs are heterogeneous and appear to differ in their presentations, similarity to existing constructs of autoimmune diseases and their natural history. To help focus the discussion regarding these complications and based on the available data on IrAEs, we propose a classification of them into three main categories (box 1). Most irAEs are self-limiting in nature and while they may have lasting clinical effects such as ongoing requirement for hormone

\footnotetext{
${ }^{1}$ Rheumatology/Immunology, Cleveland Clinic, Cleveland, Ohio, USA

${ }^{2}$ Rheumatology, Assistance Publique-Hôpitaux de Paris (AP-HP), Hôpitaux universitaires Paris-Sud - Hôpital Bicêtre, Le Kremlin Bicêtre, France

${ }^{3}$ AP-HP Université Paris Saclay, Center for Immunology of Viral Infections and Auto-immune Diseases (IMVA), Institut pour la Santé et la Recherche Médicale (INSERM) UMR 1184, Université Paris-Saclay, Le Kremlin Bicêtre, France
}

Correspondence to Dr Leonard Calabrese, Rheumatology/Immunology, Cleveland Clinic, Cleveland, OH 44106, USA; calabrl@ccf.org replacement therapy in some endocrinopathies, the inflammatory phase of these illnesses is largely self-limiting with few exceptions, with less than $10 \%$ requiring additional therapy after suppression with glucocorticoids. ${ }^{6}$ A second category is the development of a classical autoimmune disease in subjects who were predisposed. Indeed, many of these patients have been documented to have pre-existing specific autoantibodies (anti-cyclic citrullinated peptide (CCP) and they develop rheumatoid arthritis (RA), anti-SSA and they develop Sjögren's syndrome) and ICI therapy appears to act as a trigger the underlying autoimmune disease.

A third category could be that described in the current report by Braaten and colleagues, ${ }^{8}$ as they report persistent and ongoing non-specific IA. Until now, there has been a scant literature on this type of prolonged irAE, which makes this study of interest and importance. In their study published in The Annals, they interrogated a prospective observational data base from their centre of all patients referred with IA associated with ICIs and focused on those who had persistent arthritis for up to 24 months after ICIs had been stopped for treatment completion, disease progression or toxicity. With a mean follow of 9 months, they observed a remarkable 53\% had active IA at last follow-up with onequarter with active disease at 24 months. The vast majority (80\%) were treated with some dose of glucocorticoids and 24 patients required disease modifying anti-rheumtic drugs (DMARDs) including 11 with biologic disease modifying antirheumatic drugs (bDAMRDs). Interesting trends were also noted in that these patients appeared more likely to develop chronic IA if they had longer exposure to ICIs and had a history of other forms or irAEs as well. Based on these data and hints from previous smaller reported experiences, it appears that IA may be the first irAE associated with a high likelihood of developing into a chronic autoimmune and or autoinflammatory complication of ICI therapy. Clearly larger numbers of patients studied over longer time period will be required to quantify this assertion.

Based on our current understanding of IA as an irAE and its propensity for chronicity we should question what this disease truly represents, specifically asking whether it is a traditional form of IA such as RA, spodyloarthritis (SpA) or other condition or alternatively does it represent a new nosological entity in itself. As in previous reports, their patients were vastly seronegative with a tendency for more pauciarticular disease over polyarticular ones. ${ }^{3}$ In terms of etiopathogenesis,

\section{Box 1 Proposed classification of immune related adverse events (irAEs)}

Type 1

irAEs that are self-limiting in their inflammatory phase either though use of short-term immunosuppression or discontinuation of immune checkpoint inhibitors. This is the most common pattern reported. Type 1 reactions are generally non-specific in nature and not consistent with traditional classifications of autoimmune diseases.

Type 2

irAEs that appear indistinguishable for idiopathic forms of autoimmune diseases and often identified by the presence of signature autoantibodies such as antibodies to citrullinated proteins in rheumatoid arthritis, anti-acetylcholine esterase antibodies in myasthenia gravis, anti-islet cell antibodies in type 1 diabetes. ${ }^{3}$ Type 2 irAEs are rare and represent only a small proportion of all irAEs. These tend to be chronic but the natural history is still poorly characterised.

Type 3

irAEs that are chronic in their inflammatory phase and based on the current literature and the report by Brateen et $a l_{1}^{8}$ inflammatory arthritis (IA) appears to be the most common irAE to assume this clinical course. Rare reports of chronic and or relapsing colitis and pneumonitis and dermopathy ${ }^{21}$ have been reported but in general aside from IA descriptive reports are rare. 
INTEGRATED IMIMUNE RESPONSE

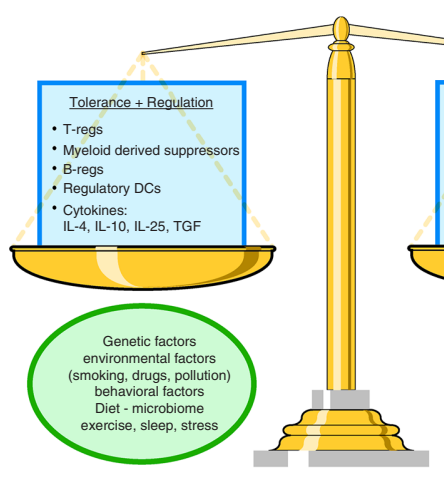

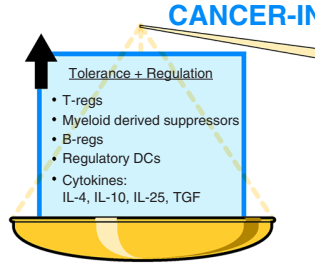
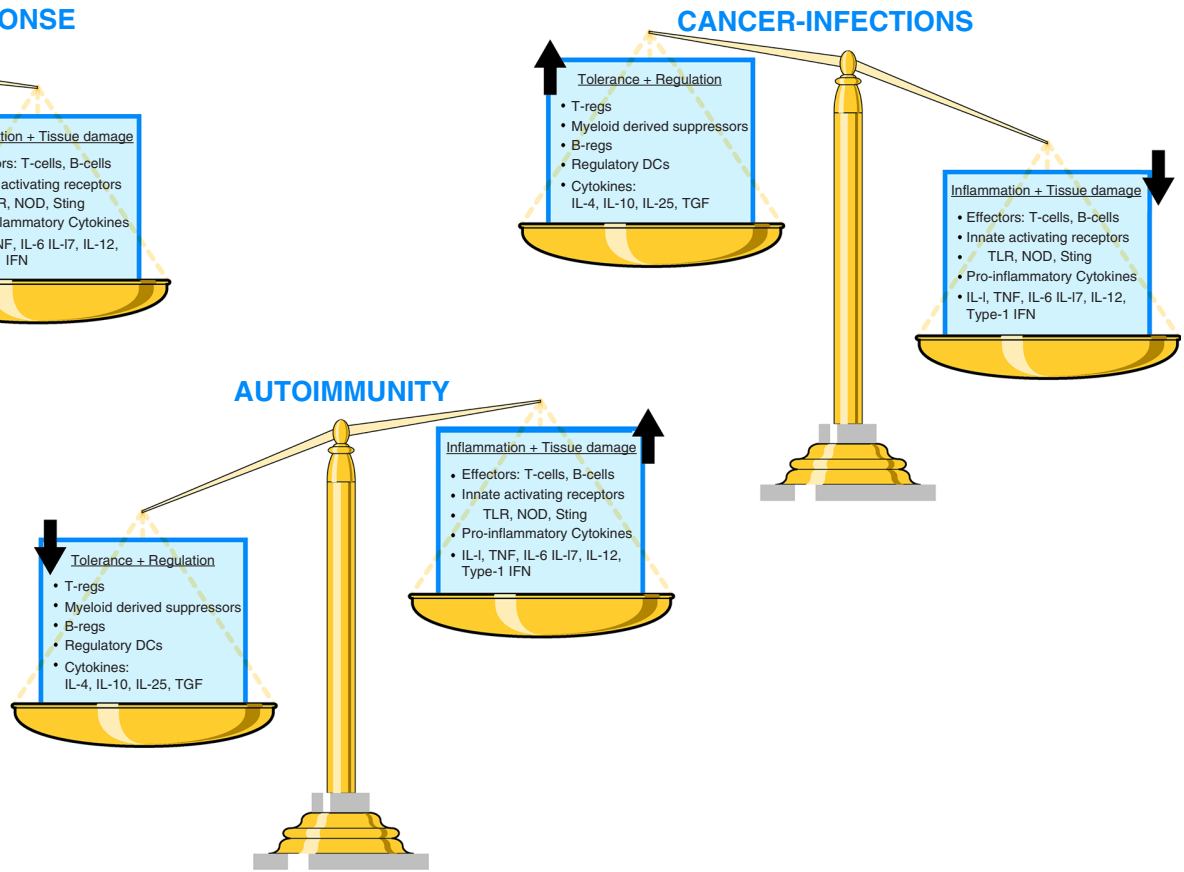

Figure 1 Immune balance. The integrated immune response in its homoeostatic state is a balance of complex tolerogenic and inflammatory forces, each contributing to optimal surveillance and patrolling functions designed to detect and dispatch danger while preserving the integrity of the host. The end product of the response is influenced by a variety of factors including the hosts genetic background and external variables and events that potentially can alter the immune system. In cancer and chronic infections there is an imbalance of depressed local-regional or systemic effector functions allowing continued growth of the tumour or persistence of infection. In autoimmunity, inflammatory effector function is overactive relative to tolerance and regulation. Both immunotherapy with ICls and immunosuppressive therapies can shift this balance, with immune checkpoint inhibitors increasing local/systemic inflammatory reactions and immunosuppressive therapies such as glucocorticoids and cDMARDS and bDMARDS and other therapies suppressing inflammation with the theoretical possibility of compromising antitumoural effects. DC, dendritic cells; IL, interleukin; NOD, nucleotide-binding oligomerisation domain-like receptors; STING, stimulator of IFN genes; TLR, toll like receptors.

the picture is far from clear but despite the preponderance of the absence of antibodies to citrullinated proteins and rheumatoid factor, a recent investigation ${ }^{9}$ has revealed that shared epitope alleles were more common in patients with ICI associated IA suggesting some commonality with RA. Clearly more investigation at the basic and translational level is needed to gain a better understanding of the condition.

The management of irAEs remains a challenge for oncologists, rheumatologists and other specialists. There is now a consensus that when clinically confronting irAEs, except in severe cases of myositis, myocarditis, pneumonitis or inflammatory bowel disease, the primary objective is to facilitate optimal treatment of the underlying cancer with ICIs or other immune based therapies whenever possible. ${ }^{10}$ The arbiter of this strategy is to balance the anti-inflammatory/immunosuppressive effects of the chosen therapy for the irAE while preserving the enhanced antitumoural effects of the immunotherapy ${ }^{11}$ (figure 1). Steroids are first line therapy used with the objective of employing the smallest effective dose. There are ongoing debates about a possible deleterious effect of steroids on cancer response to ICIs.
There are some evidence that when glucocorticoids are given at the time ICI therapy is commenced there appears to be an attenuation of their antitumoural effects. ${ }^{12}$ In addition, limited data also suggest, that at least in one complication (ie, hypohysitis), high dose glucocorticoids may compromise antitumoural immunity. ${ }^{13}$ At present however, there are no convincing data that that low doses (ie, less than $10 \mathrm{mg}$ prednisone daily) are detrimental in this setting. It also should be noted that there are mixed data on the influence of the presence of any irAE on antitumoural immunity with some suggesting enhanced effects while others no effect. ${ }^{14}$ It is interesting to note that in the current report by Braaten and colleagues, ${ }^{8}$ patients with persistent IA tended to have a better cancer prognosis than patients without persistent IA, even if most of them were treated with steroids or DMARDS. Similar beneficial effects on cancer outcome of musculoskeletal irAEs has been previously described. ${ }^{15} 16$ Further studies are necessary to determine if this new form of prolonged non-specific IA is specially associated with a better cancer prognosis.

For achieving the objective of tapering and stopping steroids, the use of DMARDs is recommended. ${ }^{17}$ In the current report by Braaten and colleagues, ${ }^{8}$ DMARDs were used in $40 \%$ of ICI-induced IA, including bDMARDs in almost half of them. In their study, use of steroids, classical synthetic disease modifying anti-rheumatic drugs (csDMARD) or bDMARD did not impact cancer outcomes. Obviously, the discussion of using DMARDs or not is particularly important in the context of this type of irAE, that is persistent non-specific IA (type 3). Overall the approach must be based on the balance between benefits and risks and may evolve depending on future studies.

The use of bDMARD to treat irAEs may raise a series of general concerns to the rheumatologist. These targeted therapies have been licensed for more than 20 years and there has been a justified anxiety on behalf of patients and providers about a possible increased risk of cancer with the class. Moreover, abatacept, a highly effective targeted therapy is the converse of ipilimumab, a very efficient ICI. However, in spite of an alert on a possible increased risk of cancer, and especially of lymphoma, with monoclonal antitumour necrosis factor (TNF) antibodies in 2006, there is no convincing evidence 
of increased risk of cancer related deaths with any bDMARD, even when used in patients with pre-existing malignancies. ${ }^{18}$

An even more provocative concept to consider is the systematic use of some bDMARDs like TNF inhibitors, tocilizumab or other targeted therapies in association with ICIs for preventing severe irAEs like severe colitis. In a preclinical study in mice, this approach was successful for preventing colitis, but also was beneficial in terms of anti-ancer effect. ${ }^{19}$ The same observation was observed in a mouse model of melanoma where the addition of anti-TNF to anti-PD1 improved cancer control and survival. ${ }^{20}$ In this study, anti-TNF attenuated overexpression of TIMP3, another checkpoint induced by anti-PD1. The beneficial effect of this association might be linked to a deleterious effect of inflammation for the action of ICIfigure 2). It is well known that a milieu of chronic inflammation may favours T cells exhaustion ${ }^{21}$ that may limit the effectiveness of ICI. Clinical studies are ongoing evaluating combination of ICIs with systemic or intratumoural use of TNF inhibitors or tocilizumab ( wwwclinincaltrials.gov NCT03293784 and NCT03588936).

In conclusion, it appears that some irAEs, especially IA, may evolve into chronic and possibly permanent inflammatory diseases that will require ongoing and perhaps lifelong immunosuppressive therapy. Rheumatologists are already being called on to participate in the management of these complex and challenging patients and a new field of irAE medicine is evolving. In this context, it is possible that, paradoxically, combining TNF or interleukin 6 inhibitors to ICI could both avoid irAEs and increase the efficacy of ICI in some specific situations. Studies to clarify what will be optimal therapy to control ongoing inflammatory diseases while preserving antitumoural immune responses are urgently needed.

Handling editor Josef S Smolen
Contributors Both LC and XM participated in all aspects of writing and reviewing the work.

Funding The authors have not declared a specific grant for this research from any funding agency in the public, commercial or not-for-profit sectors.

Competing interests None declared.

Patient consent for publication Not required.

Provenance and peer review Commissioned; internally peer reviewed.

(c) Author(s) (or their employer(s)) 2020. No commercial re-use. See rights and permissions. Published by BMJ.

\section{Check for updates}

To cite Calabrese L, Mariette X. Ann Rheum Dis 2020;79:309-311.

Received 21 October 2019

Revised 14 December 2019

Accepted 16 December 2019

Published Online First 3 January 2020

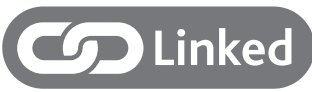

- http://dx.doi.org/10.1136/annrheumdis-2019216109

Ann Rheum Dis 2020;79:309-311.

doi:10.1136/annrheumdis-2019-216510

\section{ORCID iD}

Leonard Calabrese http://orcid.org/0000-0002-17894923

\section{REFERENCES}

1 Haslam A, Prasad V. Estimation of the percentage of US patients with cancer who are eligible for and respond to checkpoint inhibitor immunotherapy drugs. JAMA Netw Open 2019;2:e192535.

2 World Health Organization Europe. Data and statistics, 2019. Available: http://www.euro.who.int/en/healthtopics/noncommunicable-diseases/cancer/data-andstatistics

3 Calabrese LH, Calabrese C, Cappelli LC. Rheumatic immune-related adverse events from cancer immunotherapy. Nat Rev Rheumatol 2018;14:569-79.

4 Cappelli LC, Gutierrez AK, Baer AN, et al. Inflammatory arthritis and sicca syndrome induced by nivolumab and ipilimumab. Ann Rheum Dis 2017;76:43-50.

5 Calabrese C, Kirchner E, Kontzias A, et al. Rheumatic immune-related adverse events of checkpoint therapy for cancer: case series of a new nosological entity. RMD Open 2017:3:e000412.

6 Shoushtari AN, Friedman CF, Navid-Azarbaijani P, et al. Measuring toxic effects and time to treatment failure for nivolumab plus ipilimumab in melanoma. JAMA Oncol 2018;4:98-101.

7 Belkhir R, Burel SL, Dunogeant L, et al. Rheumatoid arthritis and polymyalgia rheumatica occurring after immune checkpoint inhibitor treatment. Ann Rheum Dis 2017;76:1747-50

8 Braaten TJ, Brahmer JR, Forde PM, et al. Immune checkpoint inhibitor-induced inflammatory arthritis persists after immunotherapy cessation. Ann Rheum Dis 2019. doi:10.1136/annrheumdis-2019-216109. [Epub ahead of print: 20 Sep 2019].

9 Cappelli LC, Dorak MT, Bettinotti MP, et al. Association of HLA-DRB1 shared epitope alleles and immune checkpoint inhibitor-induced inflammatory arthritis. Rheumatology 2019;58:476-80.

10 Calabrese L, Mariette X. The evolving role of the rheumatologist in the management of immunerelated adverse events (irAEs) caused by cancer immunotherapy. Ann Rheum Dis 2018;77:162-4.

11 Bucktrout SL, Bluestone JA, Ramsdell F. Recent advances in immunotherapies: from infection and autoimmunity, to cancer, and back again. Genome Med 2018;10:79.

12 Arbour KC, Mezquita L, Long N, et al. Impact of baseline steroids on efficacy of programmed cell death-1 and programmed Death-Ligand 1 blockade in patients with non-small-cell lung cancer. J Clin Oncol 2018;36:2872-8

13 Faje AT, Lawrence D, Flaherty K, et al. High-dose glucocorticoids for the treatment of ipilimumabinduced hypophysitis is associated with reduced survival in patients with melanoma. Cancer 2018;124:3706-14.

14 Postow MA, Sidlow R, Hellmann MD. ImmuneRelated adverse events associated with immune checkpoint blockade. N Engl J Med 2018;378:158-68.

15 Buder-Bakhaya K, Benesova K, Schulz C, et al. Characterization of arthralgia induced by PDantibody treatment in patients with metastasized cutaneous malignancies. Cancer Immunol Immunother 2018:67:175-82.

16 Mitchell EL, Lau PKH, Khoo C, et al. Rheumatic immune-related adverse events secondary to antiprogrammed death- 1 antibodies and preliminary analysis on the impact of corticosteroids on anti-tumour response: a case series. Eur J Cancer 2018;105:88-102.

17 Puzanov I, Diab A, Abdallah K, et al. Managing toxicities associated with immune checkpoint inhibitors: consensus recommendations from the Society for immunotherapy of cancer (SITC) toxicity management Working group. J Immunother Cancer 2017;5.

18 Regierer AC, Strangfeld A. Rheumatoid arthritis treatment in patients with a history of cancer. Curr Opin Rheumatol 2018;30:288-94.

19 Perez-Ruiz E, Minute L, Otano I, et al. Prophylactic TNF blockade uncouples efficacy and toxicity in dual CTLA-4 and PD-1 immunotherapy. Nature 2019;569:428-32.

20 Bertrand F, Montfort A, Marcheteau E, et al. Tnf $\alpha$ blockade overcomes resistance to anti-PD- 1 in experimental melanoma. Nat Commun 2017;8:2256.

21 Wherry EJ. T cell exhaustion. Nat Immunol 2011:12:492-9. 